\title{
Numerical Simulation of the Cathode Plume of a Hall Thruster
}

\author{
Maria Choi ${ }^{1}$ and Iain D. Boyd ${ }^{2}$ \\ University of Michigan, Ann Arbor, MI, 48105
}

\begin{abstract}
The plasma plume generated by the cathode of a $6-\mathrm{kW}$ laboratory Hall thruster is modeled using a hybrid particle-fluid method. The approach utilizes the direct simulation Monte Carlo method to simulate the collision dynamics of heavy particles, the particle-in-cell method to model the transport of heavy species including the effects of electrostatic fields on the ions, and a "fluid" method that solves conservation equations to compute electron properties. Two previous internal plasma simulations for a hollow cathode and discharge plasmas are utilized as input boundary conditions to the full plume simulation in this study. The simulation results are assessed through comparison with various experimental data. Since the current model neglects the magnetic fields, the effect of mapping the thruster inlet onto a magnetic field line outside the strongest regions is employed to mitigate the effect of magnetic field, which in turn results in more accurate prediction when compared with experimental data.
\end{abstract}

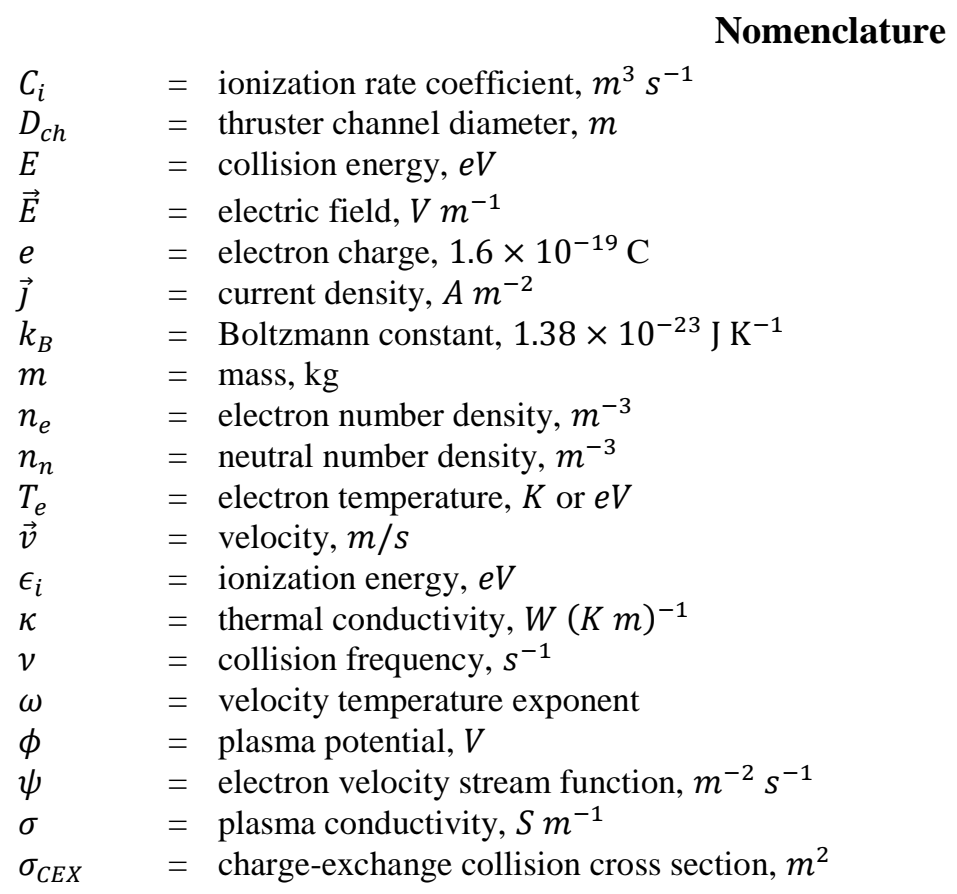

\section{Introduction}

$\mathrm{H}$ ALL-EFFECT thrusters (HETs) are efficient electric propulsion devices for spacecraft, with higher specific impulse than conventional chemical propulsion and typically higher thrust at a given power in comparison to gridded ion thrusters. ${ }^{1}$ HETs have been under development for more than 50 years, ${ }^{2}$ and over 250 HETs have been operated in space since 1971. ${ }^{1}$ Despite the great heritage, detailed physics, such as anomalous electron transport—a phenomenon common in magnetized plasma - is not yet well understood. Accurate numerical simulation is necessary

${ }^{1}$ Graduate Student, Department of Aerospace Engineering, Student Member AIAA.

${ }^{2}$ James E. Knott Professor, Department of Aerospace Engineering, Fellow AIAA.

American Institute of Aeronautics and Astronautics 
to better understand the complex plasma processes inside the thruster and in the plume of the thruster with the aim of improving thruster performance. One popular approach utilized in a Hall thruster modeling is a hybrid particle-fluid approach because it can accurately capture the bulk plasma phenomena and ion kinetics with reasonable computational expense. A previous Hall thruster modeling effort using the hybrid method includes simulations using HPHall, originally developed by Fife $^{3}$ and improved over many years. ${ }^{4-7}$ Other hybrid models are reviewed in Ref. [8-10]. These studies mainly focused on modeling the discharge plasma from inside the discharge chamber to very near-field plume of the thruster.

The goals of current study are to model the plume of a hollow cathode and assess the results using experimental data, so that more complex and important physics, such as near-wall transport and magnetic fields, can be built incrementally to better understand electron transport in the plume of the cathode of a Hall thruster. The hybrid particlefluid code MPIC, ${ }^{11}$ developed at the University of Michigan, is utilized to simulate the collision and plasma dynamics of xenon flow in the cathode plume of a 6-kW laboratory Hall thruster (H6) developed in collaboration between the Jet Propulsion Laboratory (JPL), the Air Force Research Laboratory and the University of Michigan. ${ }^{12}$ In MPIC, the collision dynamics between heavy species, i.e., neutrals and ions, are simulated using the direct simulation Monte Carlo (DSMC) $)^{13}$ method. The transport of all heavy species is simulated using the particle-in-cell (PIC) ${ }^{14}$ method, and electrons are simulated as a fluid using conservation laws.

Calculating boundary conditions at the cathode exit and discharge channel exit planes is a critical because these boundary conditions have a significant influence on the accuracy of the resulting plume structure. The present study aims to link previous internal plasma simulations of a hollow cathode and discharge channel to the plume plasma in the H6 Hall thruster, by utilizing them as boundary conditions at the cathode and discharge plasma inlets. A previous MPIC simulation utilized the hybrid Hall thruster plasma model HPHall to provide boundary conditions at the discharge channel exit to model a Hall thruster plume. ${ }^{7}$ In the past, the only heavy particles injected at the cathode inflow were xenon neutrals for simplicity, because there was no data available on the ion properties at the cathode outside of current density measurements. In the current model, full plasma conditions, i.e., ions and electron fluid, as well as neutrals are implemented at the cathode keeper exit as an inflow condition. These conditions were extracted from results provided by a global hollow cathode code, OrCa2D ${ }^{15}$ developed at the JPL. Also, the previous model did not distinguish between floating and grounded walls, and all walls were grounded. The current model accounts for the loss of high energy electrons to walls through a sheath by applying sheath-edge conditions at wall boundaries as described in the next section. The anode inflow of simulation domain in the current study is later modified to a more effective inlet geometry at the discharge channel exit, which was used previously by Huismann ${ }^{7}$ to mitigate the effect of the magnetic field in the plume. MPIC currently does not model magnetic field effects. The distinction between the present study and Ref. [7] is that the current model has more accurate boundary conditions by including a full plasma description at the cathode and sheath-edge condition at floating walls.

Section II of this paper reviews the details of the numerical methods and boundary conditions. Section III presents a series of simulation results, and a comparison of the results with experimental data. Finally, Section IV summarizes concluding remarks.

\section{Numerical Model}

\section{A. Collision Dynamics}

To simulate collisions between heavy species, i.e., $\mathrm{Xe}, \mathrm{Xe}^{+}$, and $\mathrm{Xe}^{2+}$, the DSMC method uses macroparticles that each represents a large number of real particles. Momentum exchange (MEX) and charge exchange (CEX) collisions are two basic classes of important collision mechanisms in a hollow cathode and Hall thruster plume. Collision mechanisms currently implemented in MPIC are the MEX collision between neutral-neutral and neutral-ion pairs, and the CEX collision between neutral-ion pairs. In this model, up to $\mathrm{Xe}^{2+}$ species are allowed. In the DSMC module, a list of colliding particle pairs in each cell are selected at random, regardless of their relative positions and velocities, to perform a binary collision. The collision probability is calculated at each time step, which is proportional to the product of the relative velocity between colliding particles and the total cross-section. For the neutral-neutral collisions, the variable hard sphere (VHS $)^{16}$ model is employed. The MEX cross-section for neutral-ion elastic collisions is equivalent to the CEX cross section, as demonstrated by Boyd and Dressler. ${ }^{17}$ The CEX cross-section between neutral and ion was measured by Miller et al. ${ }^{18}$ as follows:

$$
\begin{aligned}
& \sigma_{C E X}\left(X e, X e^{+}\right)=10^{-20}\left(87.3-13.6 \log _{10}(E)\right) \mathrm{m}^{2} \\
& \sigma_{C E X}\left(X e, X e^{2+}\right)=10^{-20}\left(45.7-8.9 \log _{10}(E)\right) \mathrm{m}^{2}
\end{aligned}
$$

where $E$ is collision energy in $\mathrm{eV}$. Once the collision probability is calculated, it is compared with a random number to determine whether a collision occurs or not. If a collision happens, the post-collision velocity and scattering angles

American Institute of Aeronautics and Astronautics 
are calculated using conservation of linear momentum and differential cross-section, respectively. Post-collision velocities for a MEX are assumed to follow isotropic scattering. For CEX between neutral-ion collisions, a differential cross-section is used due to a strong forward-scattering tendency. The CEX differential cross-section employed in the code was described in Ref. [18] for a Hall thruster plume.

\section{B. Plasma Dynamics}

The particle-in-cell (PIC) module tracks the motion of all heavy particles, including the effects of electrostatic fields on the ions. The charge density at each node is calculated from the distribution of the charges. The charge density is used to compute the potential at each node using a fluid electron model. Then, the electric field is calculated by spatially differentiating the electric potential. The electric field is converted into the electrostatic force, which causes acceleration on the charged particles. Quasi-neutrality is assumed to obtain the electron density from the ion number density.

The simplest fluid electron model is the Boltzmann relation, which is one of the most widely used electron models in plasma simulation:

$$
\phi=\phi_{r}+T_{e} \ln \left(\frac{n_{e}}{n_{r}}\right)
$$

where $\phi$ is electric potential, $T_{e}$ is electron temperature in $\mathrm{eV}, n_{e}$ is electron density, and the subscript $r$ indicates the reference values. The Boltzmann relation is obtained from the electron momentum equation using strong assumptions, such as that the electron fluid flow is isothermal, electron pressure obeys the ideal gas law, and magnetic fields are neglected. These assumptions are not accurate, especially in the near-field due to strong gradients.

In order to increase the level of physics as compared to the Boltzmann relation, a detailed fluid electron model was proposed. ${ }^{19}$ The detailed model solves the continuity, momentum, and energy conservation equations that describe the electron fluid at steady state, and is capable of representing more detailed descriptions for electron temperature, velocities, and plasma potential. By introducing a stream-function, $\psi$, such that $\nabla \psi=n_{e} \vec{v}_{e}=\vec{\jmath}_{e} / e$, and manipulating the equations into useful forms for numerical simulation, the following Poisson-type equations with source terms are solved:

$$
\begin{gathered}
\nabla^{2} \psi=n_{e} n_{n} C_{i} \\
\nabla \cdot(\sigma \nabla \phi)=\frac{k_{B}}{e}\left[\sigma \nabla^{2} T_{e}+\sigma T_{e} \nabla^{2} \ln \left(n_{e}\right)+\sigma \nabla \ln \left(n_{e}\right) \cdot \nabla T_{e}\right]+\frac{k_{B}}{e}\left[T_{e} \nabla \sigma \cdot \nabla \ln \left(n_{e}\right)+\nabla \sigma \cdot \nabla T_{e}\right] \\
\nabla^{2} T_{e}=-\nabla \ln \left(\kappa_{e}\right) \cdot \nabla T_{e}+\frac{1}{\kappa_{e}}\left[-\vec{\jmath} \cdot \vec{E}+\frac{3}{2} n_{e}\left(\vec{v}_{e} \cdot \nabla\right) k_{B} T_{e}\right]+\frac{1}{\kappa_{e}}\left[3 \frac{m_{e}}{m_{i}} v_{e} n_{e} k_{B}\left(T_{e}-T_{h}\right)+n_{e} n_{n} C_{i} \epsilon_{i}\right]
\end{gathered}
$$

From Eqs. (4)-(6), more detailed distributions of three fundamental plasma parameters, $\vec{v}_{e}, \phi$, and $T_{e}$ respectively, are computed using a finite element solver. ${ }^{11}$

\section{Computation Domain and Boundary Conditions}

A schematic of the 2-D axisymmetric simulation domain of the hollow cathode plume in the 6-kW laboratory Hall thruster is shown in Fig. 1. The cathode is located in the center of the thruster. For the computation of the hollow cathode plume in a Hall thruster, boundary conditions must be specified at the inflow boundaries (i.e., cathode keeper exit and discharge channel exit), along the outer edges of, and along all walls in the computational domain. Specifically, the plasma potential, electron temperature, and electron current density, and for each of the heavy species the number density, velocity, and temperature are required. Since no experimental data is available to provide all these requirements, available internal plasma simulations of a hollow cathode and inside the discharge channel were utilized to provide inflow boundary conditions.

The inflow conditions at the discharge channel exit are provided by Huismann using HPHall ${ }^{7}$ The discharge channel exit is divided into three equal line segments to represent radial variation of inflow properties, and the electron parameters at this boundary are summarized in Table 1. Since Poisson-type equations are solved, either Dirichlet (direct) or Neumann (gradient) values are required, denoted as type 1 and 2, respectively. The heavy species' inflow parameters at the discharge channel exit plane can be found in Table 3.2 of Ref. [7].

The current version of MPIC ignores the effect of magnetic fields, which is the strongest inside and in the nearfield plume of the discharge channel and diminishes rapidly as distance away from the thruster exit plane increases. Fig. 2(a) shows the HPHall computation domain, indicating the "B-field line" beyond which the magnetic field can be neglected. The left-hand side of the B-field line has high magnetic field strength, while the plume region to the right of this line has relatively weak magnetic field strength. As previously proposed by Huismann, ${ }^{7}$ the shape of anode flow inlet is modified from the discharge channel exit to the B-field line in order to neglect the region of high magnetic field strength to further improve the agreement with experimental data. The simulation case shown in Fig.

American Institute of Aeronautics and Astronautics 
1 is dubbed as the "baseline" case, and the geometry of the effective inflow case shown in Fig. 2(b) is dubbed as the "modified" case throughout this paper. The boundary conditions necessary for this model are summarized in Tables 5.1 to 5.4 in $\operatorname{Ref}[7]$.

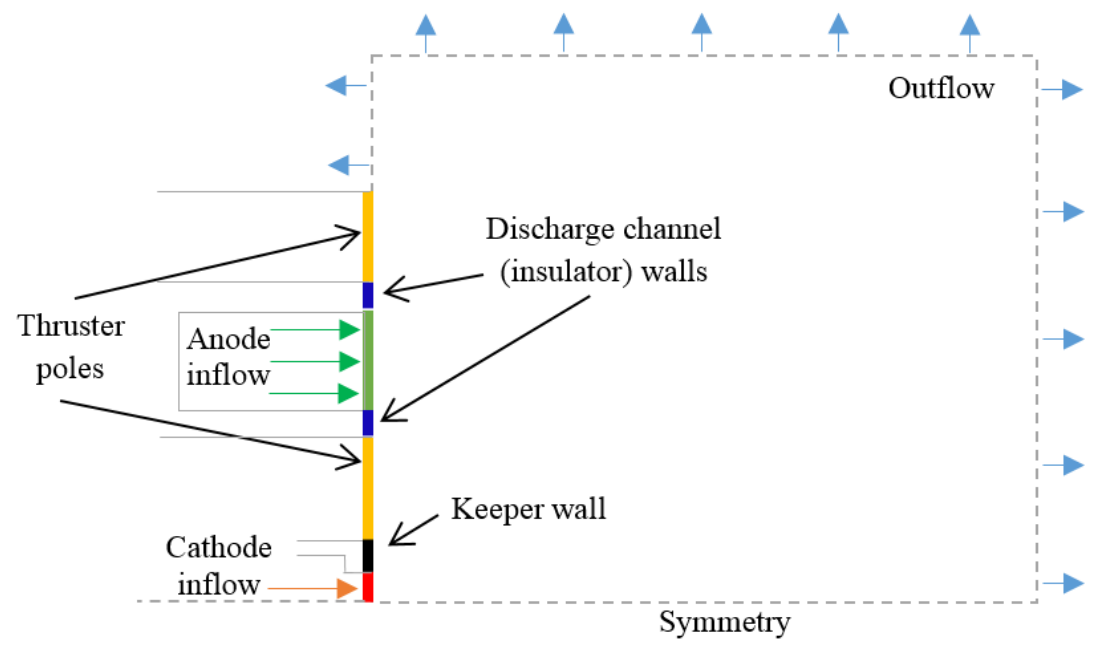

Figure 1: Two-dimensional axisymmetric computation domain of the plume of a 6-kW Hall thruster with boundaries including the symmetry line, outflows, walls, and inflows.

Table 1: Summary of inflow boundary conditions for fluid electron model. The detailed electron model requires potential, electron temperature, and stream function (Type 1: Dirichlet, and 2: Neumann)

\begin{tabular}{|c|c|c|c|c|c|c|}
\hline & Type & $\phi(V)$ & Type & $\psi\left(m^{-1} s^{-1}\right)$ & Type & $T_{e}(\mathrm{eV})$ \\
\hline Anode inflow 1 (outer) & 1 & 166 & 2 & $-5.24 \mathrm{E} 21$ & 1 & 32.5 \\
\hline Anode inflow 2 (middle) & 1 & 179 & 2 & $-7.01 \mathrm{E} 21$ & 1 & 31.1 \\
\hline Anode inflow 3 (inner) & 1 & 138 & 2 & $-6.48 \mathrm{E} 21$ & 1 & 29.8 \\
\hline
\end{tabular}

(a)

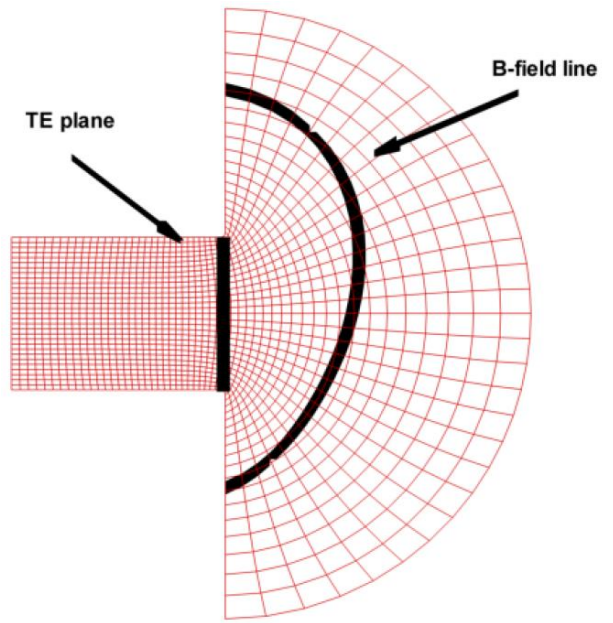

(b)

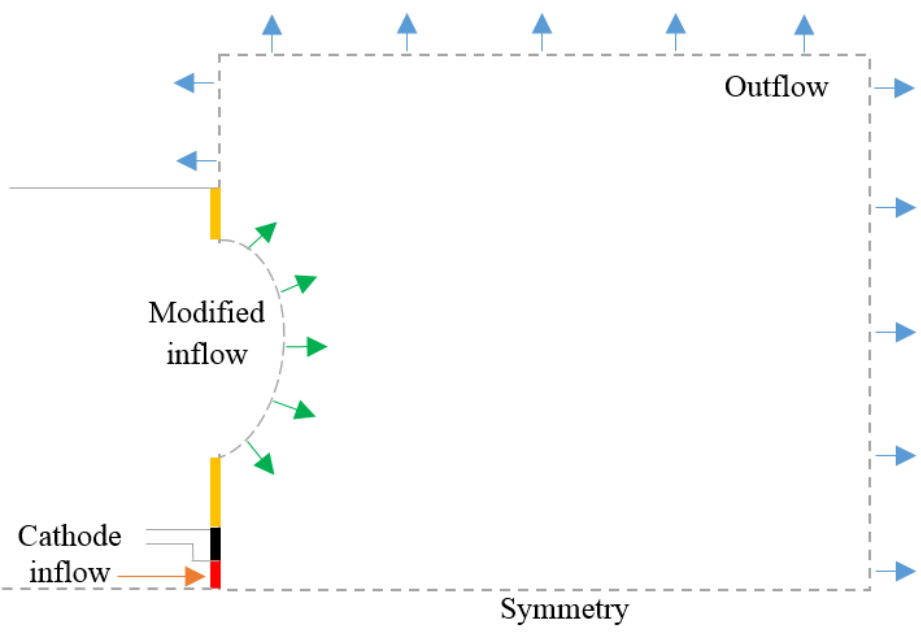

Figure 2: (a) Magnetic field line in HPHall ${ }^{7}$, and (b) new computation domain with modified inflow

The detailed electron model requires plasma potential, electron temperature, and stream function at all boundaries. The gradients of electron temperature and plasma potential normal to the boundary at the symmetry line are set to zero. The electron temperature and potential at outflow boundaries are set to constant values. Another assumption that was made in a previous MPIC simulation ${ }^{7}$ was that all walls were grounded at $0 \mathrm{~V}$. Physically, the keeper and discharge

American Institute of Aeronautics and Astronautics 
channel walls are floating, and the thruster pole pieces are grounded at $0 \mathrm{~V}$. As described below, the sheath-edge condition for floating walls are implemented in the present study. At thruster pole surfaces, the gradients of electron temperature normal to the surfaces are set to zero, and the plasma potentials are set to $0 \mathrm{~V}$. Any ions colliding with the walls are neutralized, and diffuse reflection is assumed.

As mentioned above, the plasma and neutral flow conditions at the keeper exit are extracted from $\operatorname{OrCa} 2 \mathrm{D},{ }^{15}$ which is developed at the JPL and solves conservation laws for partially-ionized gas. Figure 3 shows a contour plot of the plasma potential calculated by $\mathrm{OrCa} 2 \mathrm{D}$. The computation region includes the emitter, orifice, keeper, and plume of the cathode. The simulation was run at $20 \mathrm{~A}$ of discharge current with slight off-nominal condition for the cathode mass flow fraction of 5\% anode flow, whereas the nominal operating condition of the H6 Hall thruster is $20 \mathrm{~A}$ with the cathode mass flow fraction of 7\% anode flow. Magnetic field effect was neglected in this particular simulation. The plasma potential, electron temperature, electron current, and the velocity, temperature, and number density of heavy species are extracted at the keeper exit plane, as shown in Fig. 3, and they are used as the boundary conditions for the plume simulation using MPIC. The keeper exit plane is divided into five equal-length cells to account for the radial variation of inflow properties.

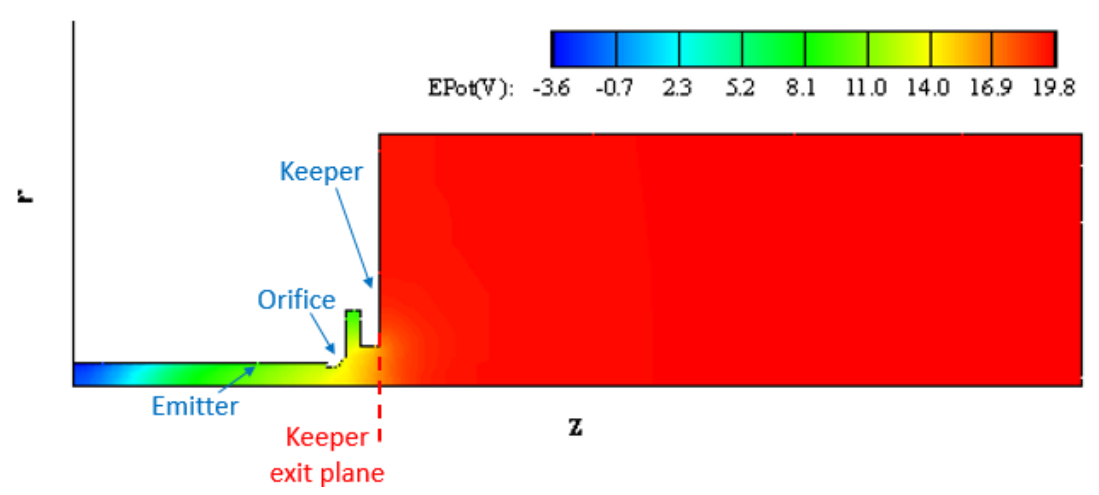

Figure 3: Plasma potential computed by OrCa2D at JPL. The computation region includes cathode emitter, orifice, keeper, and plume.

The computational domain extends to $\mathrm{z} / \mathrm{D}_{\text {thruster }}=2.8$ along the axis of symmetry from the thruster exit plane and to $\mathrm{z} / \mathrm{D}_{\text {thruster }}=1.49$ radially from the centerline of the cathode. The baseline and modified cases consist of 2282 and 2018 unstructured cells, respectively. The time step size is $4.2 \times 10^{-8}$ seconds, and a total of approximately 1.6 million particles are employed for both cases. The plasma and flow properties are sampled long after the steady state is reached.

It has been shown that the physical processes that occur inside the orifice channel and conical regions strongly influence the way the plasma evolves in the near-plume region ${ }^{15}$, which illustrates how important the conditions at the cathode inflow are to capture the physics in the near-field plume correctly. Since the current OrCa2D uses a fluid approximation in the continuum regime inside the cathode and a collisionless method from the transition regime somewhere in the cathode orifice, we eventually would like to extend our domain upstream into the cathode orifice, where the transition from continuum to rarefied flow occurs. In order to do this, the wall conditions need to be improved. MPIC is a well-developed plume simulation model for plasma where the quasi-neutral assumption is valid. Near the cathode, however, the plasma-sheath edge condition must be imposed at walls in order to capture more accurate physics. In this study, the loss of high-energy electrons to floating walls is included in the energy conservation equation of the detailed model. At the sheath-edge, the electric field is zero, and ions are accelerated to the Bohm velocity:

$$
v_{\text {Bohm }}=\sqrt{\frac{e T_{e}}{M_{i}}}
$$

Assuming there is no secondary electron emission, the sheath potential is:

$$
\phi_{s}=\frac{k_{B} T_{e}}{e} \ln \left(\frac{M}{2 \pi m_{e}}\right)^{\frac{1}{2}}
$$

where $M$ is the mass of the ion and $m_{e}$ is the electron mass. Assuming that electrons are Maxwellian, the heat flux to the wall through the sheath is calculated as:

American Institute of Aeronautics and Astronautics 


$$
q_{w, e^{-}}=\int_{-\infty}^{\infty} \int_{-\infty}^{\infty} \int_{v_{c}}^{\infty} \frac{1}{2} m_{e} n_{e}\left(v_{1}^{2}+v_{2}^{2}+v_{3}^{2}\right) v_{1}\left(\frac{m_{e}}{2 \pi k_{B} T_{e}}\right)^{\frac{3}{2}} \exp \left[-\frac{m_{e}}{2 k_{B} T_{e}}\left(v_{1}^{2}+v_{2}^{2}+v_{3}^{2}\right)\right] d v_{1} d v_{2} d v_{3}
$$

where $v_{c}=\sqrt{2 e\left|\phi_{s}\right| / m_{e}}$ is the cut-off velocity for electrons with kinetic energy greater than the sheath potential, and $v_{1}, v_{2}, v_{3}$ are velocity components in $\mathrm{x}-, \mathrm{y}-$, and $\mathrm{z}$-directions, with the $\mathrm{x}$-direction being perpendicular to the surface. By simplifying and rearranging, the flux of electron energy through the sheath is:

$$
q_{w, e^{-}}=\frac{1}{4} n_{e}\left(\frac{8 k_{B} T_{e}}{\pi m_{e}}\right)^{\frac{1}{2}} \exp \left(-\frac{e \phi_{s}}{k_{B} T_{e}}\right)\left(e \phi_{s}+2 k_{B} T_{e}\right)
$$

The energy flux is multiplied by the computational cell area and electron number density entering the sheath. This term is incorporated into the electron energy equation as a heat loss to the wall.

\section{Results and Comparisons with Measurements}

The hybrid particle-fluid plume model, MPIC, described above is applied to simulate the 6-kW laboratory Hall thruster. Simulations are performed using two computational regions as shown in Figs. 1 and 2(b) as a baseline and modified cases, respectively. In this section, a series of simulation results are presented, accompanied by comparisons made with measurements taken by Sekerak, ${ }^{20}$ Jameson, ${ }^{21}$ and Reid. ${ }^{22}$ The thruster was operated at the following nominal operating conditions: discharge voltage of $300 \mathrm{~V}$, discharge current of $20 \mathrm{~A}$, anode flow rate of $20 \mathrm{mg} / \mathrm{s} \mathrm{of}$ xenon, and cathode flow rate measuring $7 \%$ of the anode flow. For the experimental data provided by Sekerak, the background pressure during the thruster operating was $1 \pm 0.1 \times 10^{-5}$ Torr, the plasma potential and electron temperature measurements were taken using high-speed dual Langmuir probes, and the uncertainties associated with these measurements were approximately $25 \%$. For the data measured by Jameson, the background pressure during the thruster operating was $1.7 \times 10^{-5}$ Torr. The plasma potential and electron temperature were measured with an emissive probe and cylindrical Langmuir probe, respectively, with uncertainties of $\pm 1 \mathrm{~V}$ for both quantities. The relative neutral number density was measured with an optical probe, which uses optical emission spectroscopy to infer the local xenon neutral density. The relative neutral density is a local measurement of how the injected neutral gas atoms are dispersed in the system and provides information on where they are depleted by ionization. ${ }^{21}$ The uncertainty in neutral density measurement was $2 \%$. The ion current density measurements were performed by Reid ${ }^{22}$ using two Faraday cup probes. The experimental data by Reid had approximately the same background chamber pressure during the thruster operation as Jameson. The uncertainty associated with the near-field probe measurements is $\pm 10 \%$, whereas the far-field probe measurements have $\pm 0-50 \%$ on the integrated beam current. Simulations using MPIC are performed with two different background gas pressures to compare with these experimental data sets accordingly.

Comparisons are made between the two thruster discharge inlet cases - the baseline case and the modified inflow case-as discussed in the previous section. The thruster inlet for the baseline case is at the discharge channel exit plane, while the modified inflow condition is applied at the magnetic field line geometry as shown in Fig. 2. Both cases utilize full particle and fluid properties at the keeper exit plane extracted from OrCa2D ${ }^{15}$ These boundary conditions have a significant influence on the accuracy of the resulting plume structure.

In Fig. 4(a), contour plots of the plasma potential for the baseline (top) and modified cases (bottom) are compared. As expected, the plasma potential fields of the two cases are very different. The baseline case shows higher potential in the near-field plume, while the potential of modified case is not as high. Electron temperature is compared in Fig. 4(b). The baseline case shows high electron temperature at the discharge channel exit and has steep gradient over the entire domain. Both the potential and temperature gradients predicted by the modified case is much lower than the baseline case. In Fig. 4(c), both cases show that the ion current density is high near the discharge channel exit, and then decreases rapidly downstream along the discharge channel centerline. The peak observed at the centerline of the cathode in the baseline case is not physical and is probably due to numerical issue. The neutral number density contours are compared in Fig. 4(d). The modified case predicts higher neutral density in the near-field plume of cathode centerline than the baseline case. The neutral density at cathode keeper exit is in the order of $10^{19} \mathrm{~m}^{-3}$, and it decrease away from the cathode centerline with spherical shape for both cases, baseline case having steeper gradient than the modified case.

American Institute of Aeronautics and Astronautics 
(a)

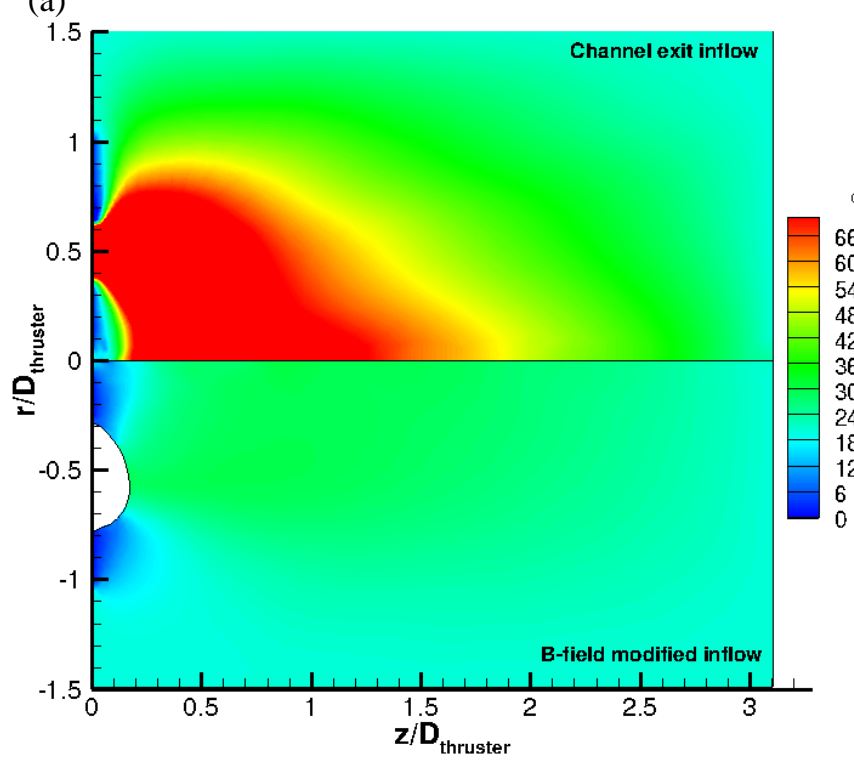

(c)

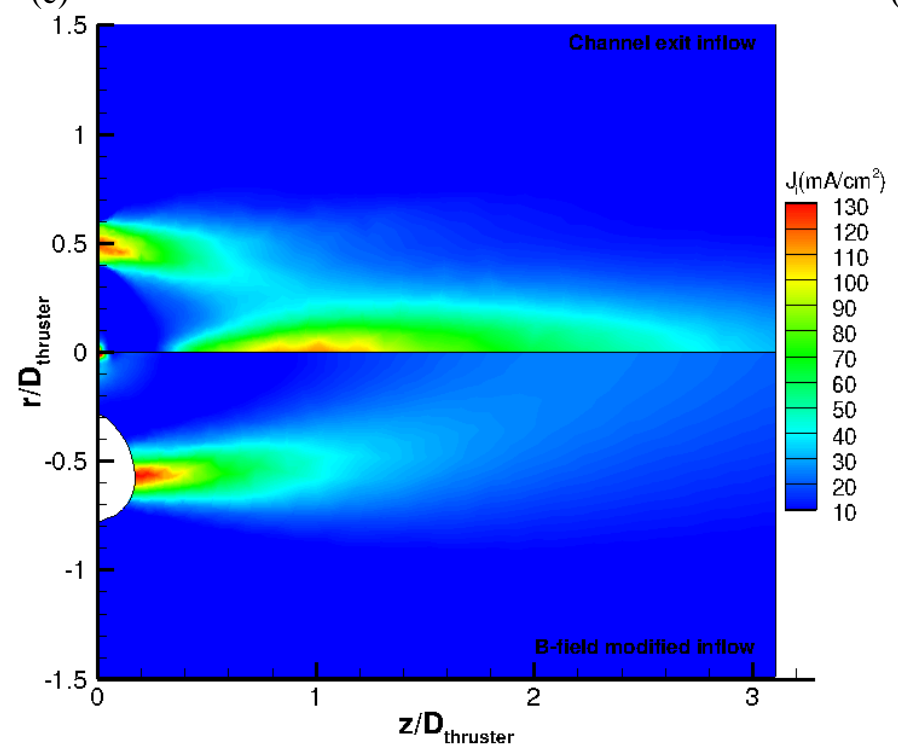

(b)

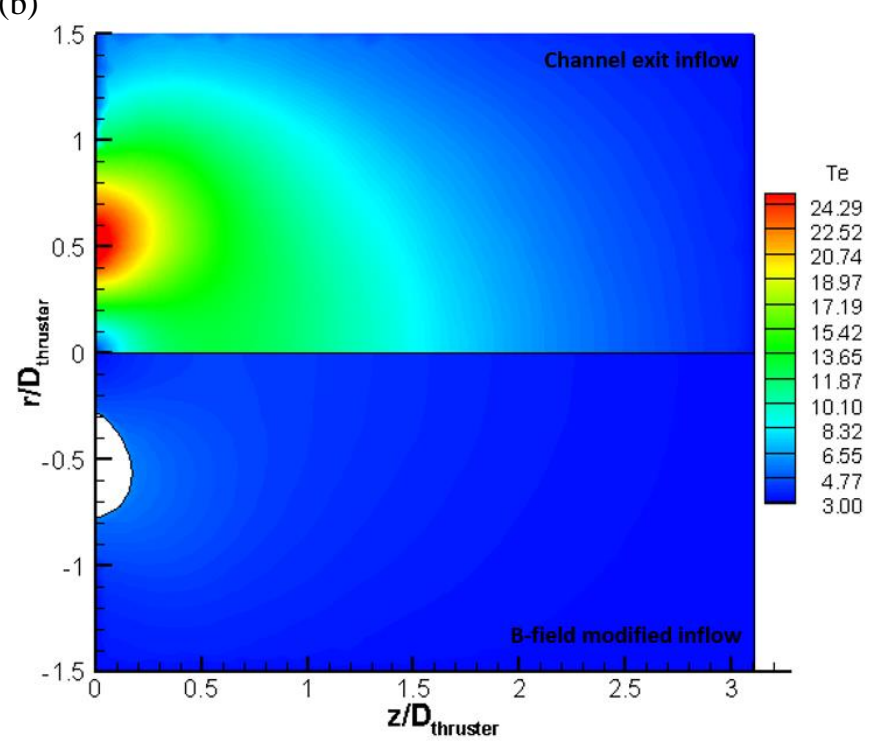

(d)

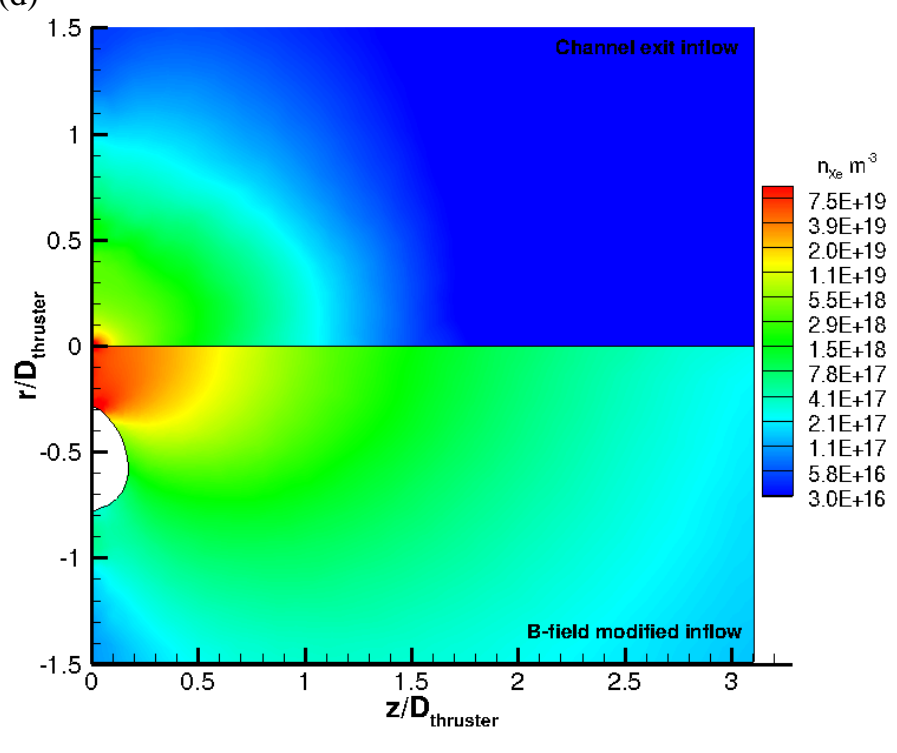

Figure 4: Contour plots of (a) plasma potential, (b) electron temperature, (c) ion current density, and (d) neutral number density for the baseline inflow (top) and modified inflow (bottom) cases.

More quantitative comparisons of the plasma potential, electron temperature, ion current density, and plasma number density along the axial direction are shown in Figs. 5(a)-(d). Figs. 5(a), (b), and (d) are extracted along the cathode centerline, while Fig. 5(c) is along the discharge channel centerline. Axial displacements are normalized by thruster diameter $\mathrm{D}_{\text {thruster. }}$ Plasma potential is over-predicted by approximately $50 \mathrm{~V}$ for the baseline case-labeled as channel exit flow-in the near-field plume, while the modified case predicts plasma potential profile well. The potential gradient predicted by the modified case is smaller than the baseline case. Fig. 5(b) shows that the baseline case over-estimates electron temperature by approximately $5 \mathrm{eV}$ at $\mathrm{z} / \mathrm{D}_{\text {thruster }}=0.4$. These over-estimations in potential and electron temperature are primarily due to the fact that magnetic field effects are ignored. While the modified case maintains reasonable electron temperature in the far-field plume, it does not capture the peak observed in the measurement. This may be due to ignoring the heat flux to the thruster pole pieces, which will be investigated in the future. In Fig. 5(c), the ion current density for the baseline case is slightly under-predicted although it gives the correct trend shown by the measurement. The modified case predicts the current density well. In Fig. 5(d), plasma number density from both simulation cases show decreasing profile in the very near-field plume, which is caused by sudden 
expansion in the plume. The density gradient for modified case is lower than the baseline case in the near-field. While the measurement reaches a steady value further downstream, the simulation cases show a slow decrease in the density.
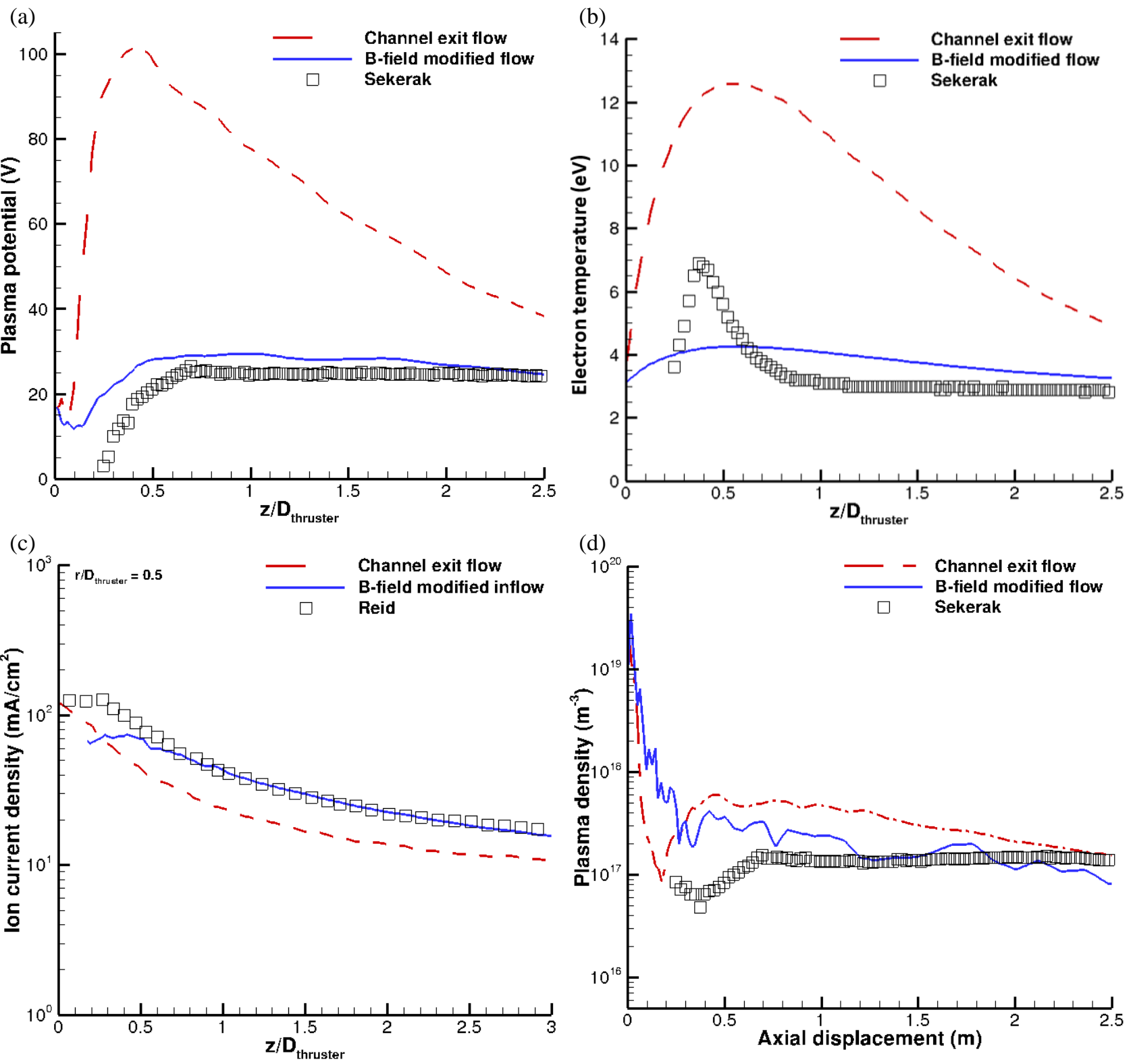

Figure 5: Comparisons of (a) plasma potential, (b) electron temperature, (c) ion current density, and (d) plasma number density between the baseline (labeled as channel exit flow) and modified cases with experimental data. ${ }^{20-22}$ Figures (a), (b), and (d) are along the cathode centerline, and (c) is along the center of discharge channel (r/D $\mathbf{D}_{\text {thruster }}$ $=0.5)$

Further comparisons between the experimental data and simulation results for radial profiles of plasma potential, ion current density, and neutral number density are shown in Figs 6-8, respectively, where (a) and (b) are near-plume results and (c) and (d) are far-plume results. Figs. 6(a)-(d) show the radial profile of plasma potential, which is again over-estimated by the baseline case. The modified case shows better agreement with the measured data. It is noticable from Figs. 6(a)-(c) that the experimental data shows the lowest radial potential value at the cathode centerline $\left(\mathrm{r} / \mathrm{D}_{\text {thruster }}\right.$ $=0$ ), while both simulation results do not predict the low potential measured. This may also be due to the fact that the current simulation by MPIC, as well as OrCa2D, does not account for the magnetic field. In the far-field as shown in Fig. 6(d), the modified case shows constant plasma potential profile similar to the measured data. 
(a)

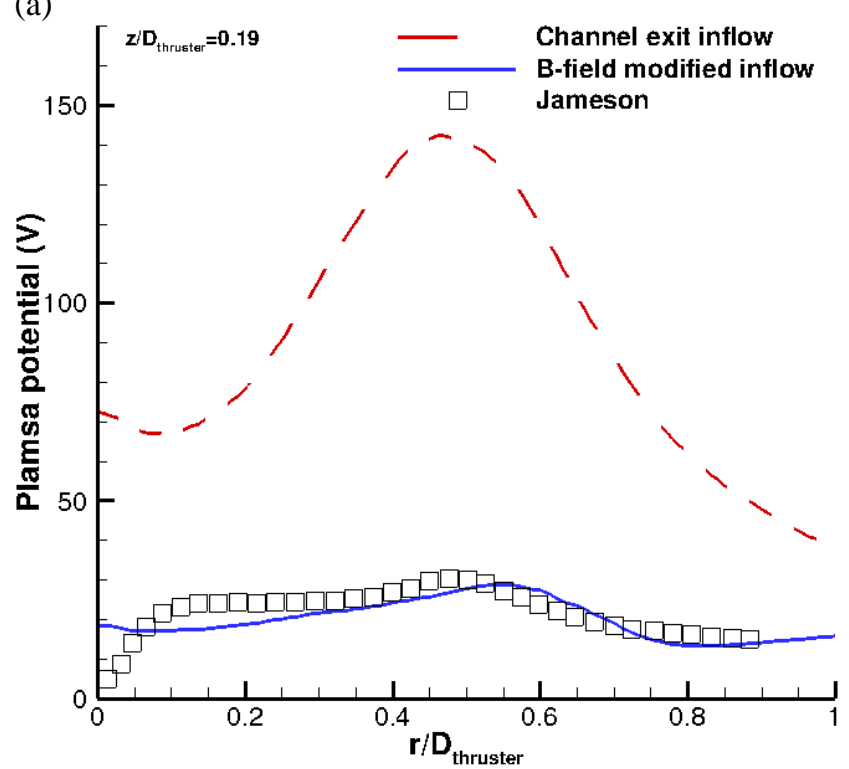

(c)

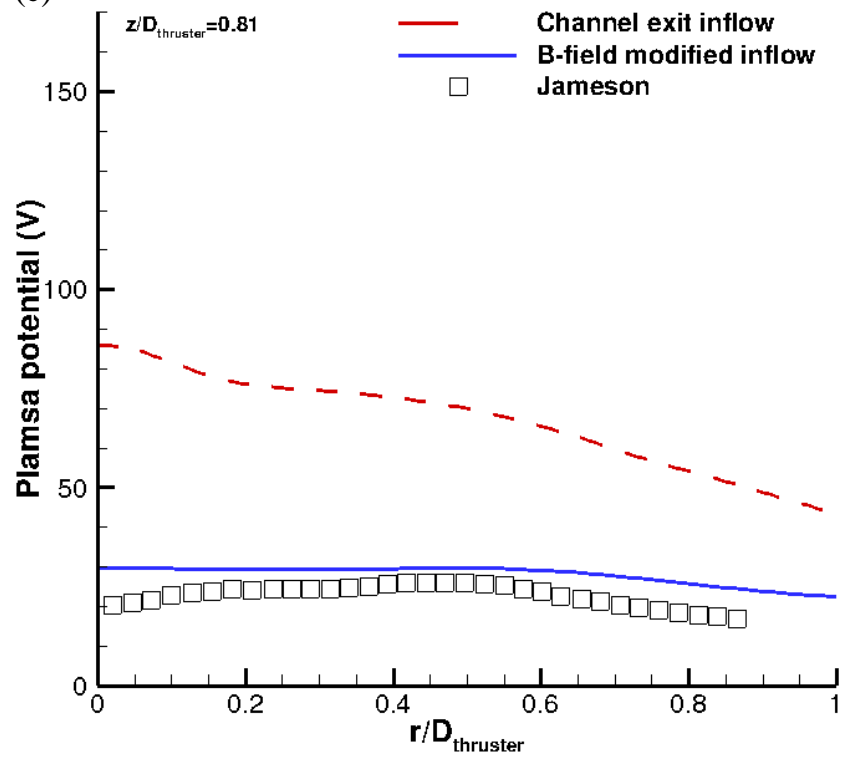

(b)

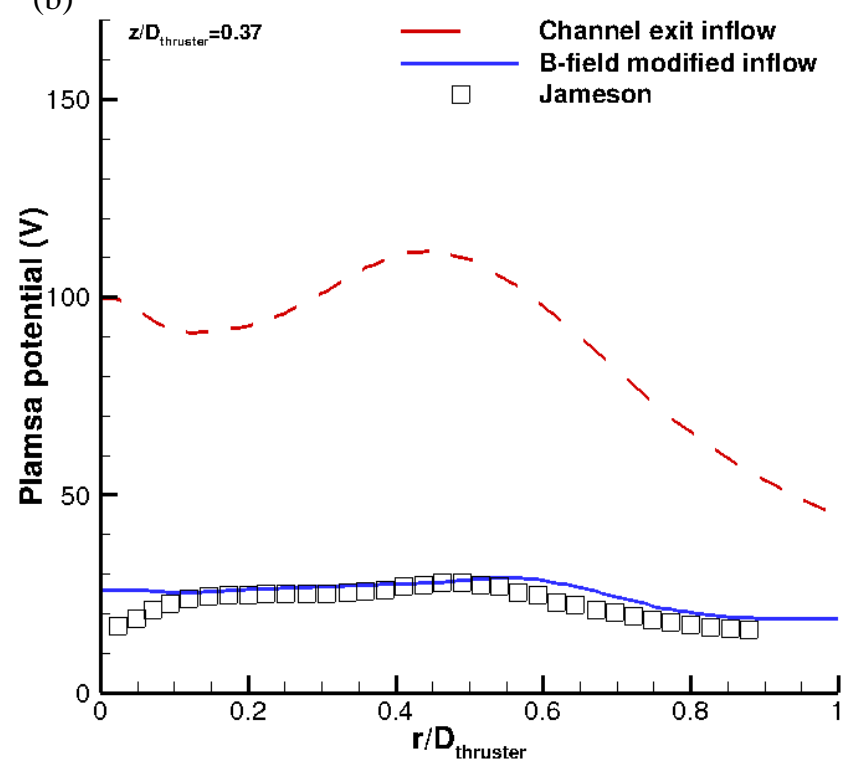

(d)

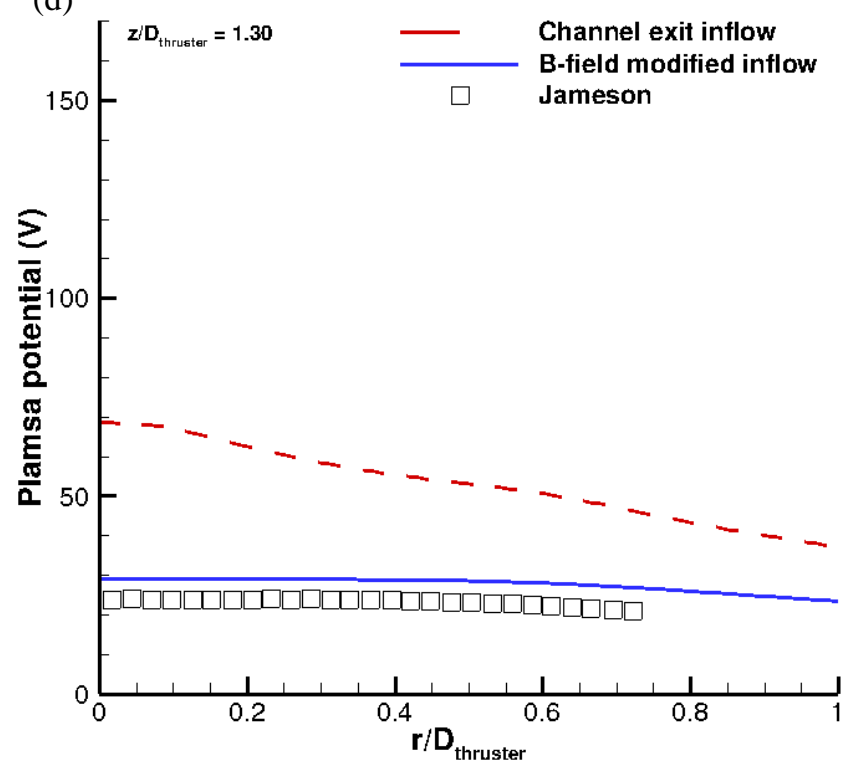

Figure 6: Comparison of plasma potential along radial displacement of the thruster at axial locations of $\mathrm{z}=$ (a) $3 \mathrm{~cm}$, (b) 6 $\mathrm{cm}$, (c) $13 \mathrm{~cm}$, and (d) $21 \mathrm{~cm}$, with measurement by Jameson. ${ }^{21}$

In Fig. 7, ion current density in the radial direction at different axial locations are compared with experimental data by Reid. ${ }^{22}$ Fig. 7(a) shows the baseline case only because $\mathrm{z} / \mathrm{D}_{\text {thruster }}=0.08$ is so closed to the thruster exit plane that the radiap profile is cut-off due to the modified inflow geometry. In the near-field plume, the baseline case underpredicts ion current density near the centerline of the thruster and at large radial position as shown in Fig. 7(a). However, the parabolic profile near the discharge channel exit is predicted well. Fig. 7(b) is still the near-field, and the radial profile of ion current density predicted by the baseline case starts to deviate from the measured profile near the discharge channel exit. The agreement between the baseline case and measurement diverges further downstream as shown in Figs. 7(c) and (d). On the other hand, the result predicted by the modified case is under-estimated ion current density near the centerline of the thruster but agrees well with the profile shape at larger radius starting from near the discharge channel exit, as shown in Fig. 7(b). Similar observation is shown in the far-field plume profiles in Fig. 7(c) and (d) where the modified case matches the measured ion current density better at large radii.

American Institute of Aeronautics and Astronautics 
(a)

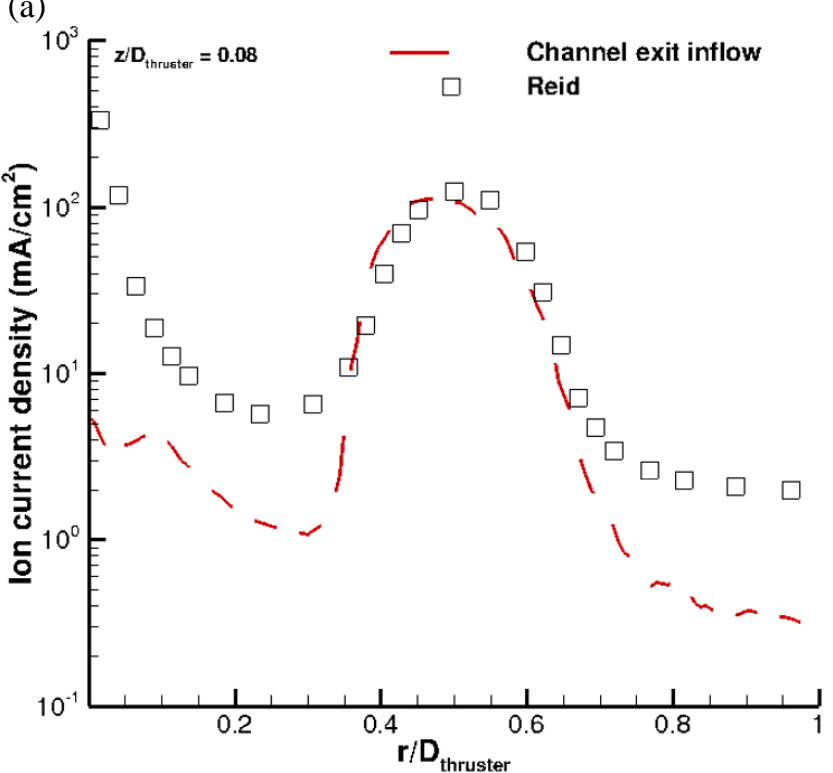

(c)

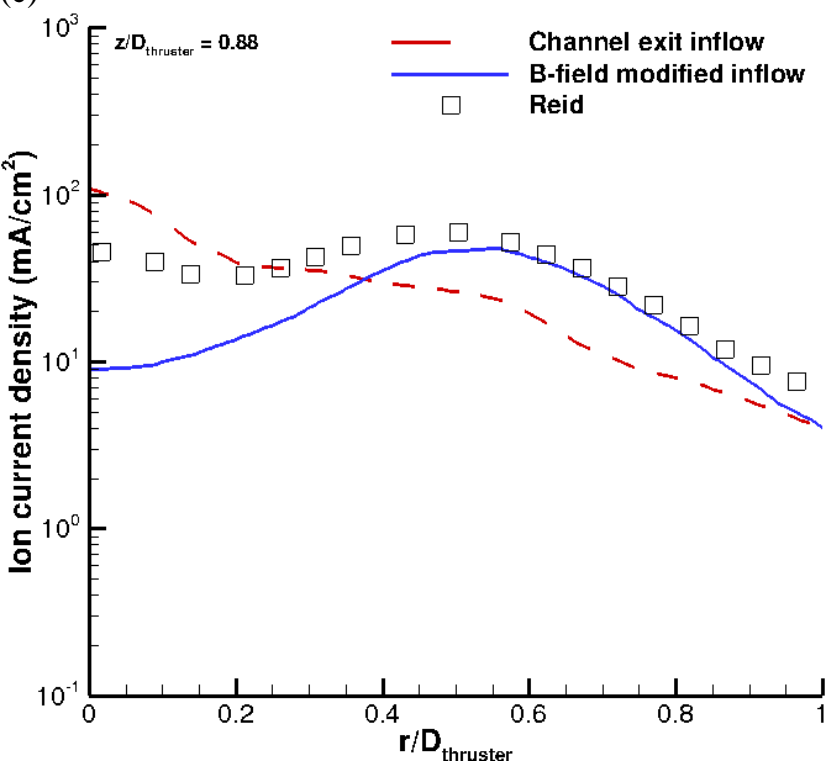

(b)

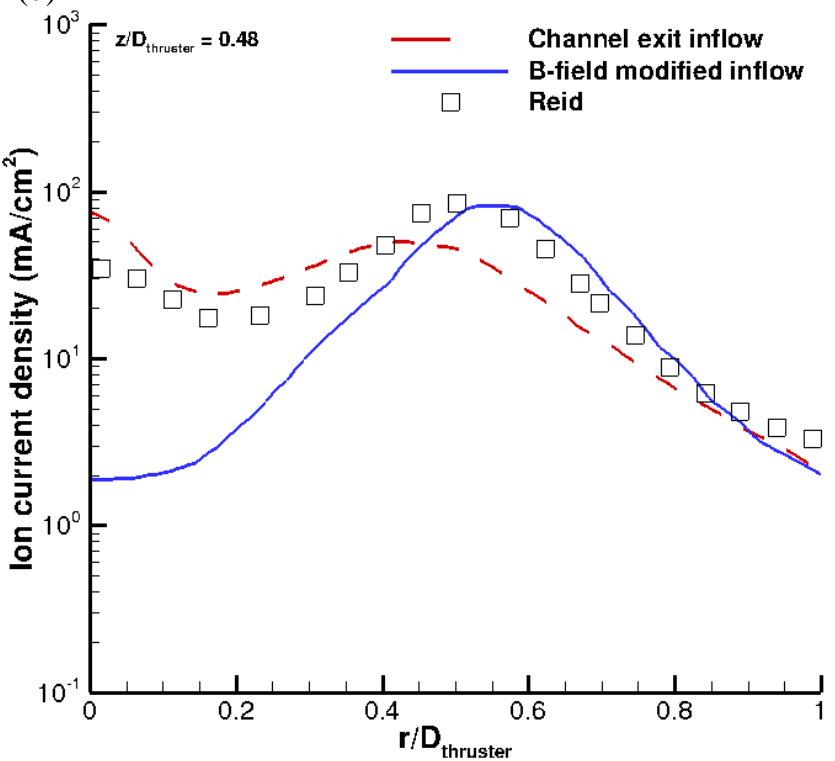

(d)

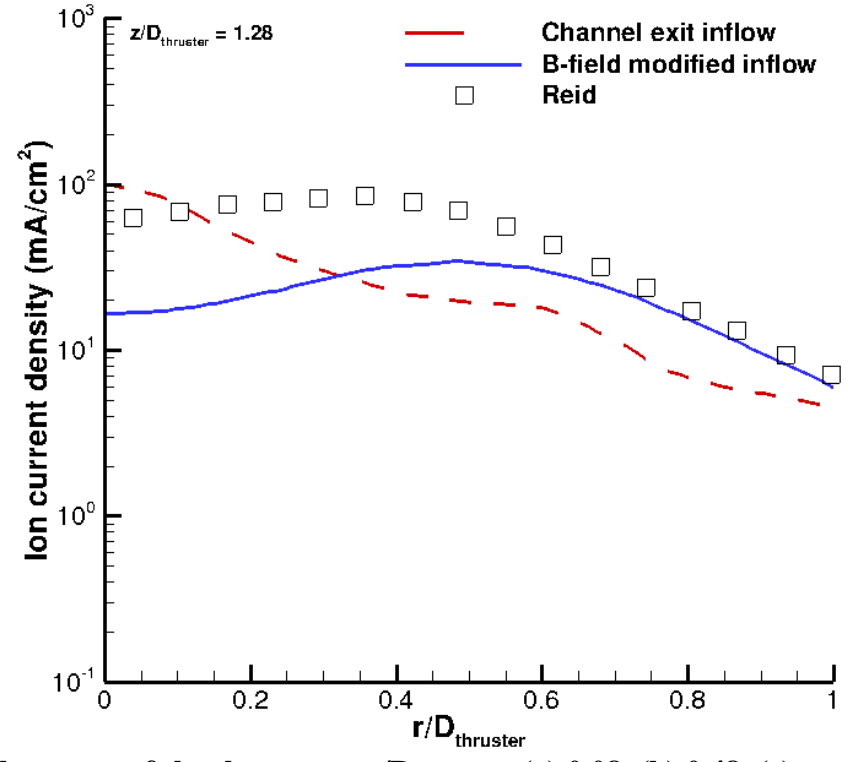

Figure 7: Comparison of ion current density along radial displacement of the thruster at $\mathrm{z} / \mathrm{D}_{\text {thruster }}=(\mathrm{a}) \mathbf{0 . 0 8 , ( b )} 0.48,(\mathrm{c})$ 0.88 , and (d) 1.28 measured by Reid ${ }^{21}$

In Fig. 8, the neutral number density is compared with experimental data of Jameson. ${ }^{21}$ As previously mentioned, the measurement represents a relative neutral density, not the absolute number density, so it is scaled by a constant value to match the lowest data point to the background pressure. Therefore, the comparison is focused more on the general trend of the profile shape than the magnitude. The near-field plume measurements shown in Figs. 8(a) and (b) show the peaks at the cathode centerline $(\mathrm{r} / \mathrm{D}=0)$ and near the discharge channel exit. Both the baseline and modified case results do not show the peaks observed in the experimental data. The simulation results show higher peak near the cathode centerline and the neutral density decreases radially outward. The measured relative neutral density profile further away from the thruster shows a smaller peak along the discharge channel centerline $(r / D=0.5)$ and a flattened profile at the cathode centerline, as shown in Fig. 8(c). The measurement shows that the relative neutral density eventually flattens out at the far-field plume, as shown in Fig. 8(d). Both simulation results do not predict any peaks, but show almost flat neutral density in the radial direction with slightly greater magnitude along the centerline of the cathode than at larger r/D.

American Institute of Aeronautics and Astronautics 
(a)

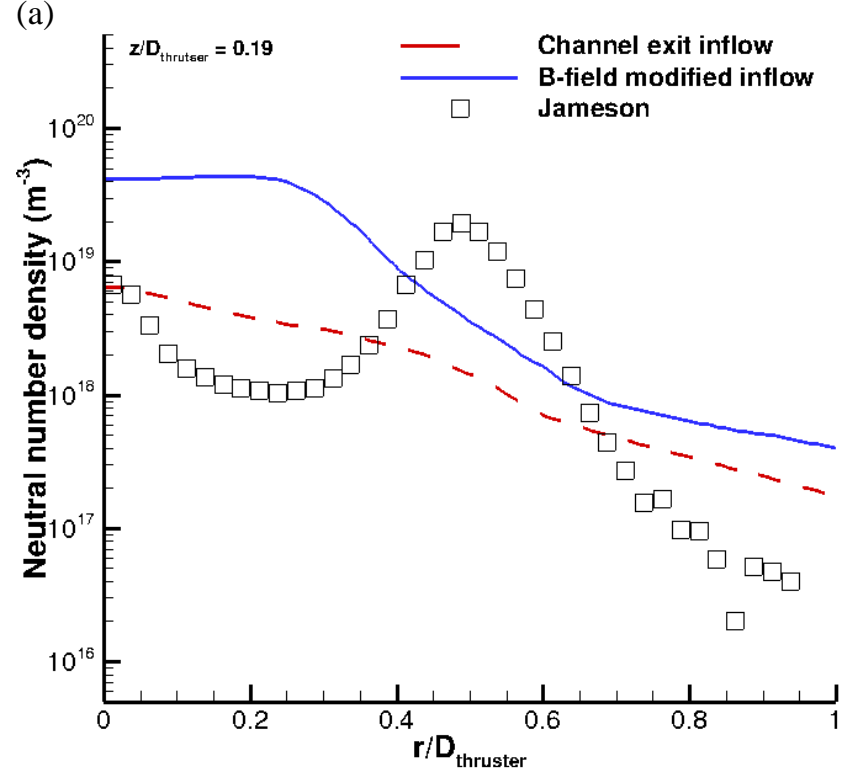

(c)
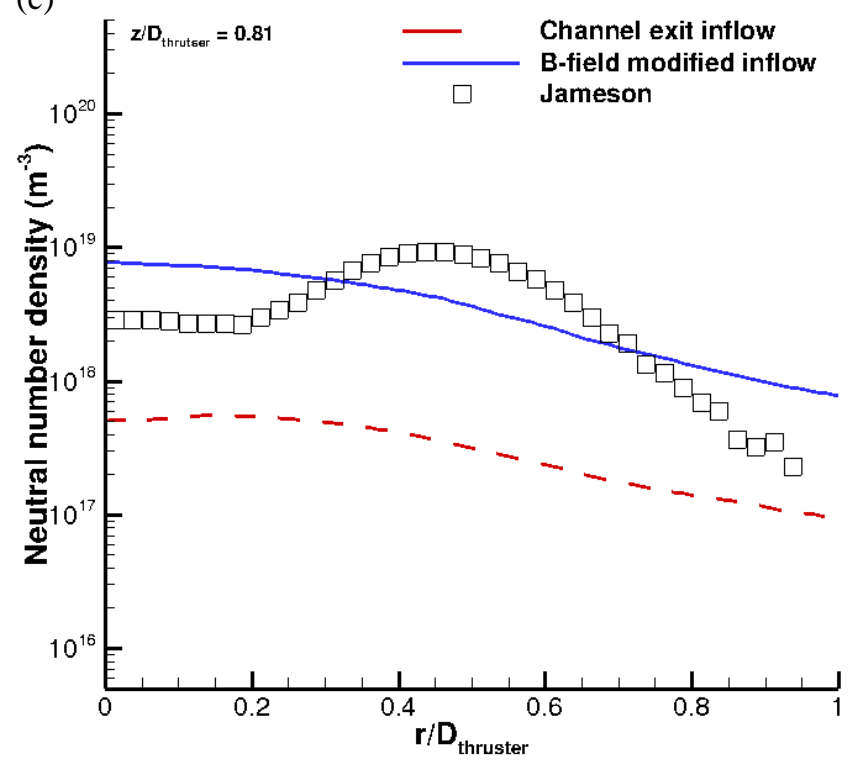

(b)

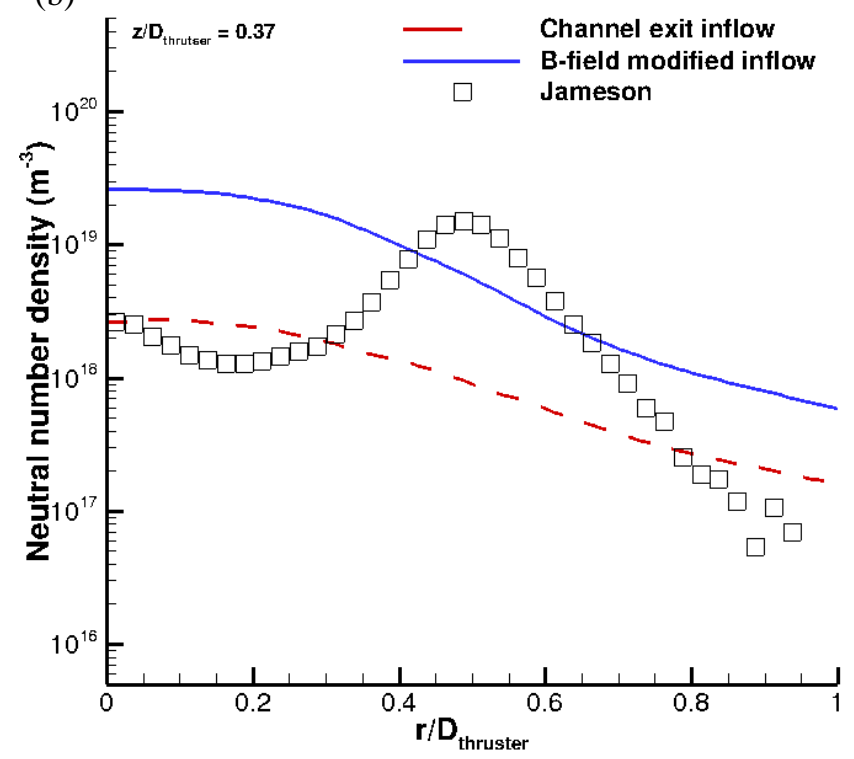

(d)

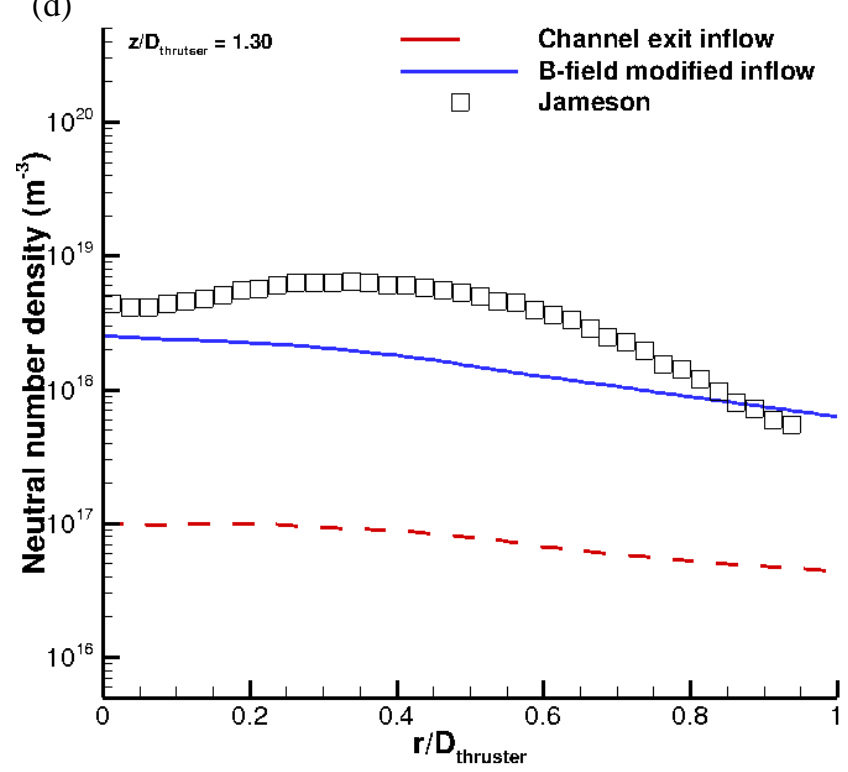

Figure 8: Comparison of neutral number density along radial displacement of the thruster at axial locations of $\mathrm{z}=(\mathrm{a}) 3 \mathrm{~cm}$, (b) $6 \mathrm{~cm}$, (c) $13 \mathrm{~cm}$, and (d) $21 \mathrm{~cm}$, with measurement by Jameson ${ }^{21}$

\section{Conclusions}

Motivated by previous modeling work, the current MPIC simulations attempted to model more accurate cathode plume physics by improving boundary conditions at the cathode inflow utilizing detailed flow conditions from an OrCa2 $\mathrm{D}^{14}$ simulation and at floating wall with sheath-edge conditions. Two different anode geometries were simulated: 1) the baseline case with inflow at the discharge channel exit, and 2) the modified case that effectively eliminates the effect of magnetic field which is neglected in current MPIC simulation. The modified case provided better agreement with experimental data in general than the baseline case. It is confirmed that the effect of magnetic field cannot be ignored in the cathode plume simulation. To address the remaining disparities, future work will involve adding more accurate physics to the simulations, such as magnetic fields and heat loss to the thruster pole surfaces.

American Institute of Aeronautics and Astronautics 


\section{Acknowledgments}

The authors would like to thank Dr. Richard Hofer and Dr. Ioannis Mikellides of the Jet Propulsion Laboratory (JPL), California Institute of Technology, and Dr. Michael McDonald and Dr. Michael Sekerak of the University of Michigan for providing data files for the simulation input and measurement comparisons. This work is supported by NASA Office of the Chief Technologist's Space Technology Research Fellowship (NSTRF), Grant \#NNX11AN77H.

\section{References}

${ }^{1}$ Goebel, D. M., and Katz, I. (2008). Fundamentals of electric propulsion: ion and Hall thrusters (Vol. 1). John Wiley \& Sons.

${ }^{2}$ Kim, V., Popov, G., Arkhipov, B., Murashko, V., Gorshkov, O., Koroteyev, A., and Tverdokhlebov, S. (2001). Electric propulsion activity in Russia. IEPC Paper, 5, 2001.

${ }^{3}$ Fife, J. M., "Hybrid-PIC Modeling and Electrostatic Probe Survey of Hall Thrusters," Ph.D. Thesis, Aeronautics and Astronautics, Massachusetts Institute of Technology, 1998.

${ }^{4}$ Parra, F. I., Ahedo, E., Fife, J. M., and Martinez-Sanchez, M., "A two-dimensional hybrid model of the Hall thruster discharge," Journal of Applied Physics, Vol. 100, No. 2, 2006.

${ }^{5}$ Escobar, D. and Ahedo, E., "Improved electron formulation for a Hall thruster hybrid model," 42 ${ }^{\text {nd }}$ AIAA/ASME/SAE/ASEE Joint Propulsion Conference \& Exhibit, No. 2006-4326, Sacramento, California, USA, July 2006.

${ }^{6}$ Hofer, R. R., Katz, I., Mikellides, I., Goebel, D. M., Jameson, K. K., Sullivan, R. M., and Johnson, L. K., "Efficacy of Electron Mobility Models in Hybrid-PIC Hall Thruster Simulations," 44th AIAA/ASME/SAE/ASEE Joint Propulsion Conference \& Exhibit, No. 2008-4924, Hartford, Connecticut, USA, July 2008.

${ }^{7}$ Huismann, T. D. (2011). Improving hall thruster plume simulation through refined characterization of near-field plasma properties. Ph.D. Dissertation, University of Michigan Ann Arbor.

${ }^{8}$ VanGilder, D. B., Boyd, I. D., \& Keidar, M. (2000). Particle simulations of a Hall thruster plume. Journal of Spacecraft and Rockets, 37(1), 129-136.

${ }^{9}$ Sommier, E., Scharfe, M. K., Gascon, N., Cappelli, M. A., \& Fernandez, E. (2007). Simulating plasma-induced Hall thruster wall erosion with a two-dimensional hybrid model. Plasma Science, IEEE Transactions on, 35(5), 1379-1387.

${ }^{10}$ Hagelaar, G., Bareilles, J., Garrigues, L., and Boeuf, J. P., “Two Dimensional Model of a Stationary Plasma Thruster,” Journal of Applied Physics, 91, p. 5592, 2002.

${ }^{11}$ Cai, C. (2005). Theoretical and numerical studies of plume flows in vacuum chambers. Ph.D. Dissertation, University of Michigan Ann Arbor.

${ }^{12}$ Haas, J. M., Hofer, R. R., Brown, D. L., Reid, B. M., and Gallimore, A. D. (2007, May). Design of the H6 Hall Thruster for High Thrust/Power Investigation. In 54th JANNAF Propulsion Meeting, Denver, CO (pp. 14-17).

${ }^{13}$ Bird, G. A. (1994). Molecular gas dynamics and the direct simulation of gas flows.

${ }^{14}$ Birdsall, Charles Kennedy, and Allan Bruce Langdon. Plasma Physics via Computer Simulation. Adam Hilger, U.K., 1991.

${ }^{15}$ Mikellides, I. G., Katz, I., Goebel, D. M., and Polk, J. E. (2005). Hollow cathode theory and experiment. II. A two-dimensional theoretical model of the emitter region. Journal of Applied Physics, 98(11), 113303.

${ }^{16}$ Bird, G. A. (1981). Monte-Carlo simulation in an engineering context. Progress in Astronautics and Aeronautics, 74, 239255.

${ }^{17}$ Miller, J. S., Pullins, S. H., Levandier, D. J., Chiu, Y. H., and Dressler, R. A. (2002). Xenon charge exchange cross sections for electrostatic thruster models. Journal of Applied Physics, 91(3), 984-991.

${ }^{18}$ Boyd, I. D., and Dressler, R. A. (2002). Far field modeling of the plasma plume of a Hall thruster. Journal of Applied Physics, 92(4), 1764-1774.

${ }^{19}$ Boyd, I. D., and Yim, J. T. (2004). Modeling of the near field plume of a Hall thruster. Journal of Applied Physics, 95(9), $4575-4584$

${ }^{20}$ Sekerak, M., McDonald, M., Hofer, R., and Gallimore, A. (2013, March). Hall thruster plume measurements from high-speed dual langmuir probes with ion saturation reference. In Aerospace Conference, 2013 IEEE (pp. 1-16). IEEE.

${ }^{21}$ Jameson, K. K. (2008). Investigation of hollow cathode effects on total thruster efficiency in a $6 \mathrm{~kW}$ Hall thruster. Ph.D. Dissertation, University of California Los Angeles.

${ }^{22}$ Reid, B. M. (2009). The influence of neutral flow rate in the operation of Hall thrusters. Ph.D. Dissertation, University of Michigan Ann Arbor.

American Institute of Aeronautics and Astronautics 